\title{
HPV-Mediated (p16-Positive) Oropharyngeal Cancer Clinical Distant Metastasis TNM Finding v8
}

National Cancer Institute

\section{Source}

National Cancer Institute. HPV-Mediated (p16-Positive) Oropharyngeal Cancer Clinical

Distant Metastasis TNM Finding V8. NCI Thesaurus. Code C132878.

A clinical finding about one or more characteristics of HPV-mediated (p16-positive)

oropharyngeal cancer, following the rules of the TNM AJCC v8 classification system as they pertain to distant metastases. 